\title{
Evaluation of Stress, Anxiety, Depression, and Sleep Disorders in Medical Students of Hamadan University of Medical Sciences, Iran, during the COVID-19 Pandemic
}

\author{
Zahra Miri' $^{\text {(i) }}$, Zahra Razavi2,*(i), Shaghayegh Mohammadi ${ }^{1}$ \\ ${ }^{1}$ Intern, School of Medicine, Hamadan University of Medical Sciences, Hamadan, Iran \\ ${ }^{2}$ Professor, Department of Pediatrics, School of Medicine, Hamadan University of Medical Sciences, Hamadan, Iran \\ * Corresponding Author: Zahra Razavi, Department of Pediatrics, School of Medicine, Hamadan University of Medical \\ Sciences, Hamadan, Iran. Email: razavizahra@yahoo.com.au
}

Received: 18.10 .2020 Accepted: 28.01 .2021

\section{How to Cite this Article:}

Miri Z, Razavi Z, Mohammadi S. Evaluation of Stress, Anxiety, Depression, and Sleep Disorders in Medical Students of Hamadan University of Medical Sciences, Iran, during the COVID-19 Pandemic. Avicenna $J$ Clin Med. 2021; 27(4): 232-238. DOI: $10.29252 / \mathrm{ajcm} .27 .4 .238$

\section{Abstract}

Background and Objective: One of the high-risk groups for infection with coronavirus disease 2019 (COVID-19) is considered medical students. In this regard, this study was designed and carried out to evaluate the status of stress, anxiety, depression, and sleep disorders in the medical students of Hamadan University of Medical Sciences, Hamadan, Iran, during the COVID-19 pandemic.

Materials and Methods: This cross-sectional descriptive study was conducted in the first half of 2020 on medical students (clerks and interns). The information required for the study, including demographic characteristics, study year, accommodation in a dormitory or home, and levels of anxiety, stress, depression, and sleep disorders were online collected by completing the standard Depression, Anxiety, and Stress Scale questionnaire and 7-Item Insomnia Severity Index, respectively. The data were analyzed using SPSS software (version 16). A p-value of less than 0.05 was considered statistically significant.

Results: A total of 257 medical students were surveyed $55.6 \%$ of whom were female. The mean value of students' age was $24.09 \pm 1.61$ years. In this study, $171(66.6 \%)$ participants were interns and $105(40.9 \%)$ were living in dormitories. Moreover, $62.3 \%(n=160)$ of the students were in contact with COVID-19 patients. After the pandemic, about $28.4 \%, 17.1 \%, 21.8 \%$, and $20.6 \%$ of the participants were depressed, stressed, anxious, and with sleep disorders, respectively. There was no statistically significant relationship among the variables of demographics, accommodation in the dormitory, and their study year with stress, anxiety, depression, and insomnia.

Conclusion: The incidence of psychological complications, including anxiety, stress, depression, and insomnia, in the medical students of Hamadan University of Medical Sciences during the COVID-19 outbreak is high. In critical situations, such as the recent pandemic, appropriate prevention, early interventions, and access to mental health services are essential to prevent dysfunction and maintain the mental health of vulnerable groups.

Keywords: Anxiety, COVID-19, Depression, Medical Students, Sleep Dis orders, Stress 


\title{
بررسى وضعيت استرس، اضطراب، افسردى و اختلال خواب در دانشجويان يزشكى دانشعاه علوم يزشكى هملان طى هملَّيرى كوويد 19
}

\author{
زهرا ميرى ' (iD) ، زهرا رضوى (iD) شقايق محمدى'

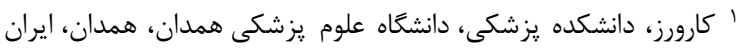

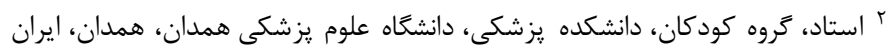
razavizahra@y ahoo.com.au : نويسنده مسئول: زهرا رضوى، گروه كود كان، دانشكده يزشكى، دانشكاه علوم يزشكى همدان، همدان، ايران. ايميل

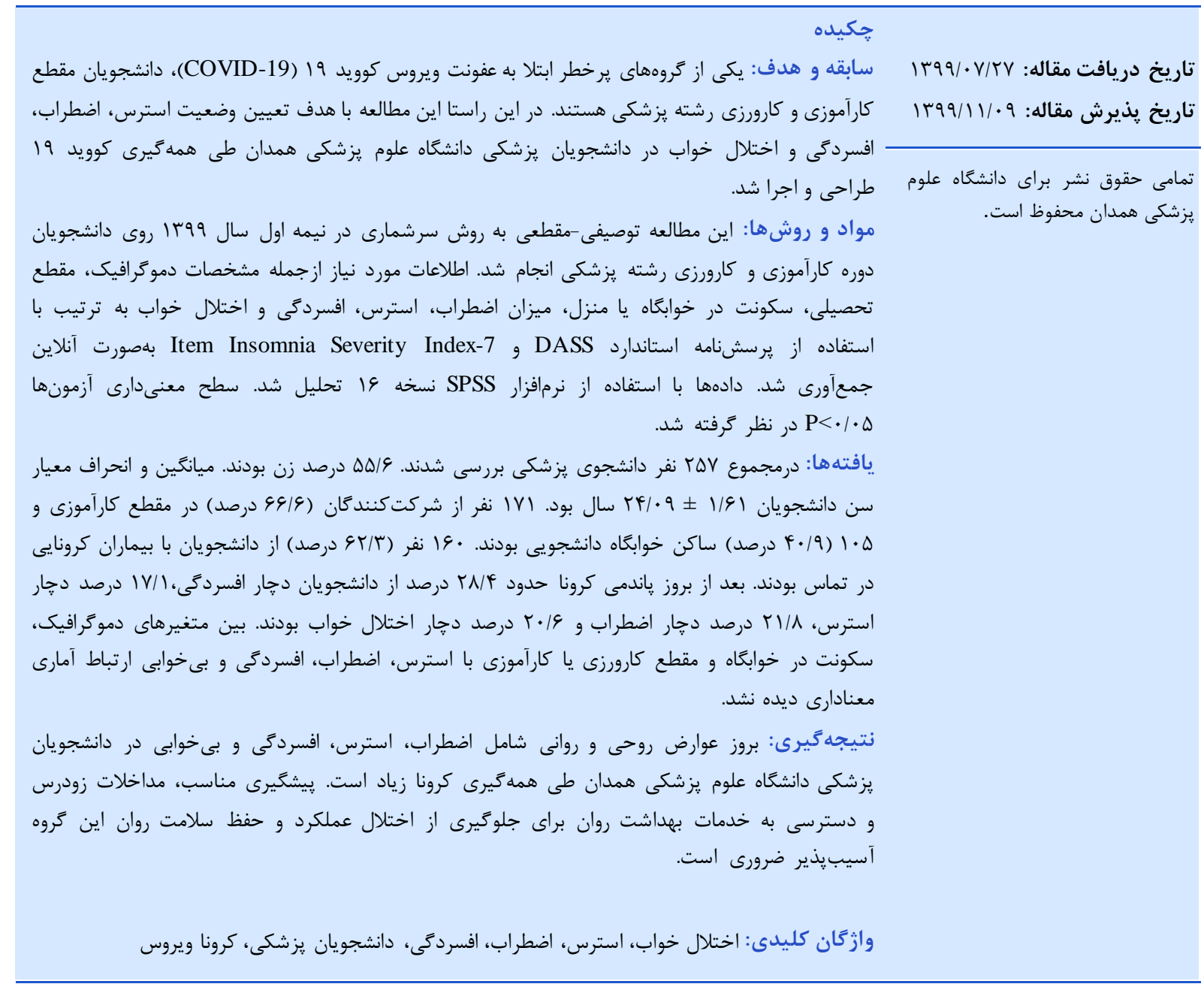

مقلدمه

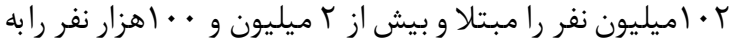

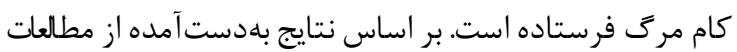

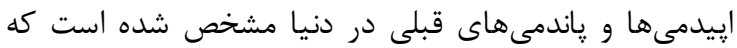

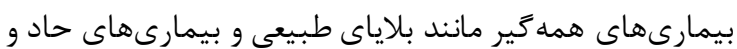

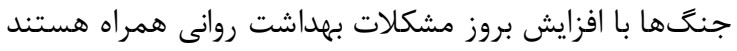

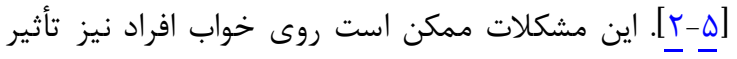

ابتلا به ويروس كرونا 9 ا م ب (COVID-19) كه قبلاً بهعنوان

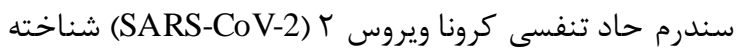

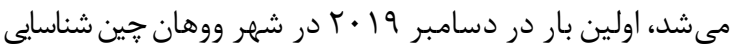

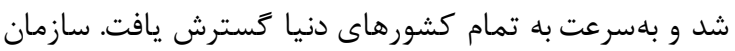

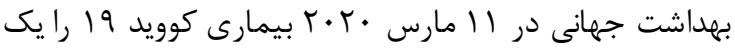

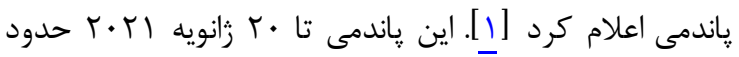




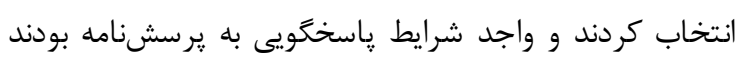

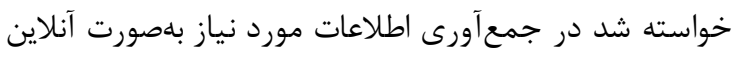

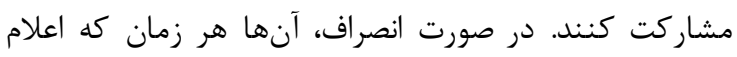

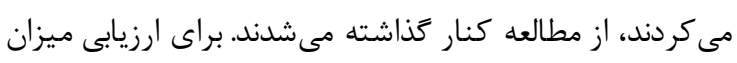

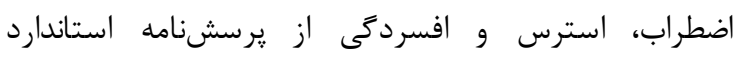
و Depression, Anxiety and Stress Scale DASS-21

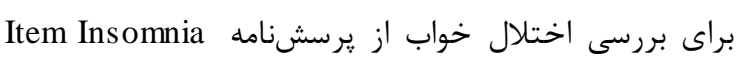
ير Severity Index-7

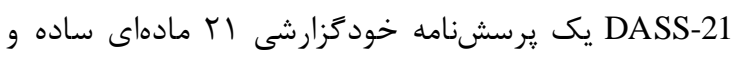

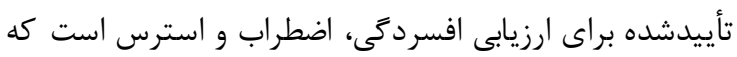

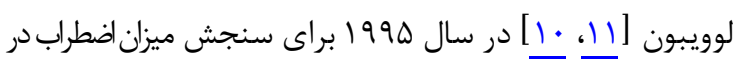

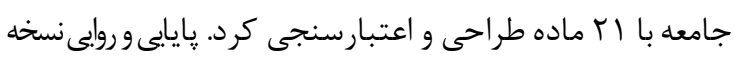

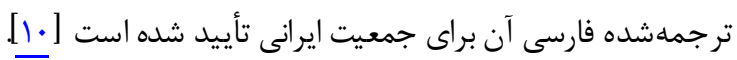

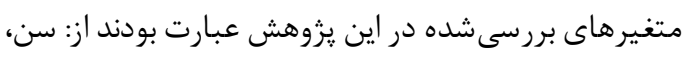

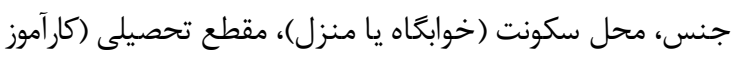

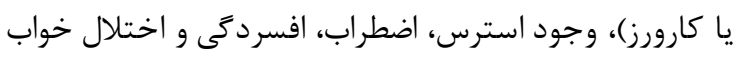

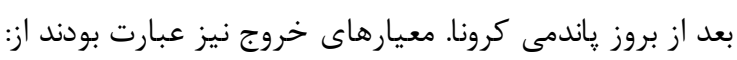

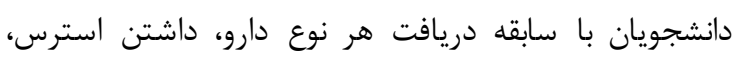

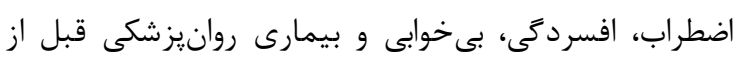

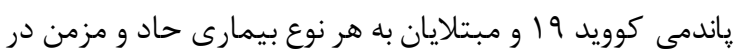
زمان تكميل برسش كاندامه.

شر كت كنند

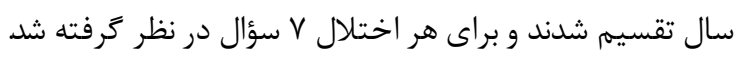

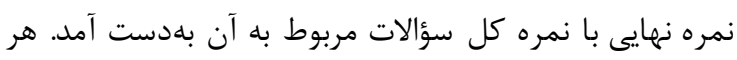

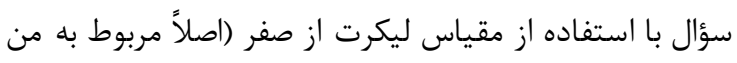

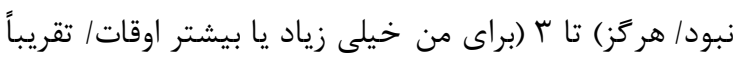

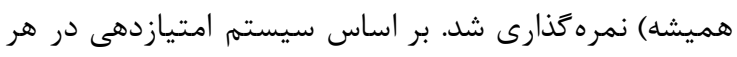

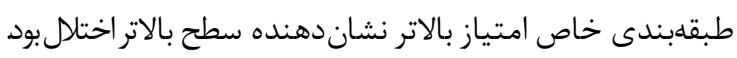

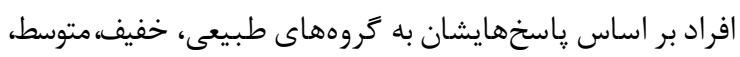

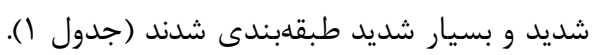

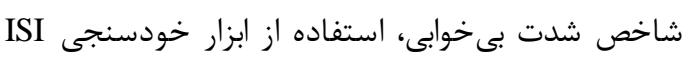

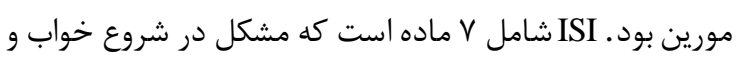

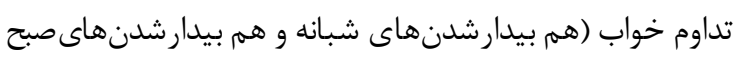

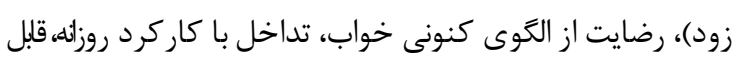

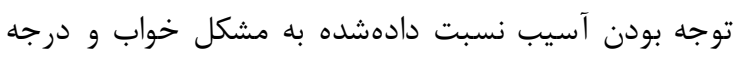

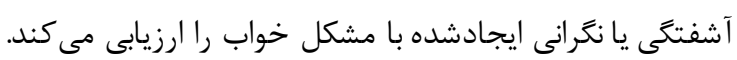

جدول ا: طبقهبندى شدت عوارض روحى و روانى

\begin{tabular}{|c|c|c|c|}
\hline استرس & اضطر اب & افسردَى & شدت \\
\hline$\cdot-1 f$ & $\cdot-V$ & - -9 & عادى \\
\hline$|\omega-1|$ & $1-9$ & $1 \cdot-1 \mu$ & خفيف \\
\hline $19-r \Delta$ & $1 \cdot-\mid f$ & $\mid f-r$. & متوسط \\
\hline זr-4G & $10-19$ & $41-4 R$ & شديد \\
\hline سז+ & $+r$. & $+r \Lambda$ & بسيار شديد \\
\hline
\end{tabular}

منفى داشته باشد [ب].

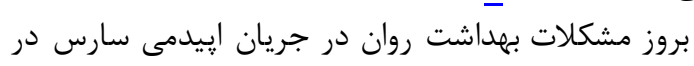

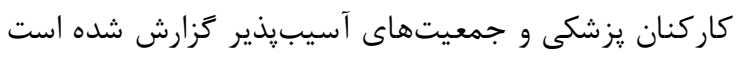

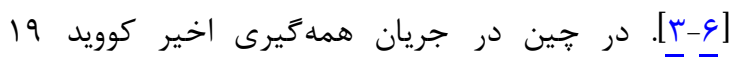

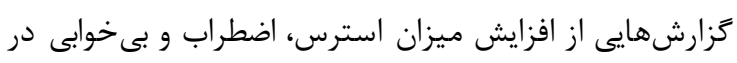

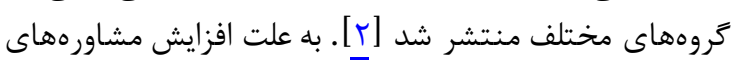
روانيزشكى و درخواست كمك زياد مردم بلهويزه كاركنان

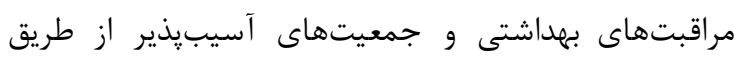

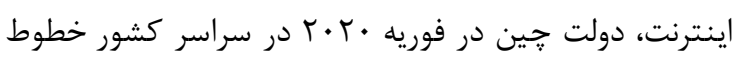

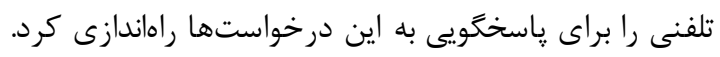

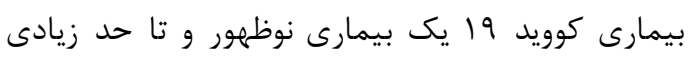

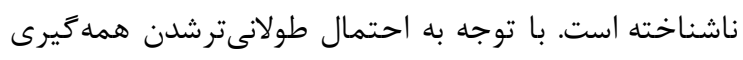

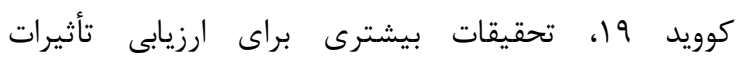

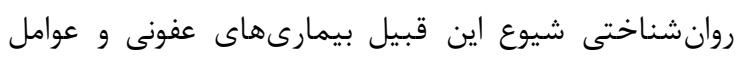

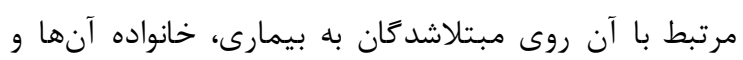

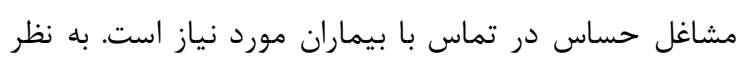

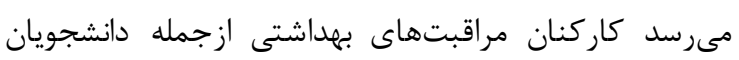

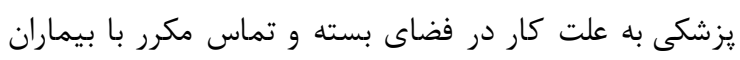

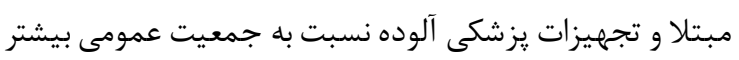

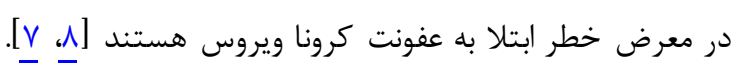

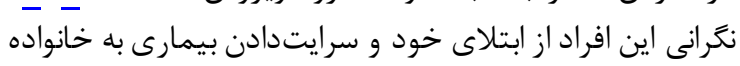

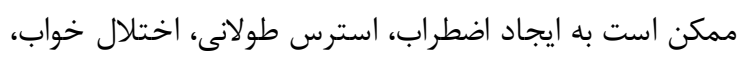

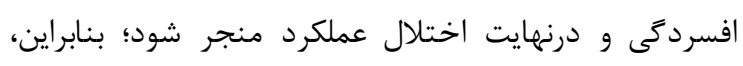

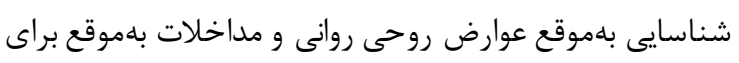

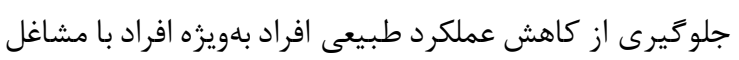

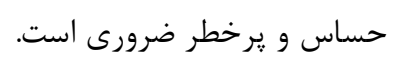

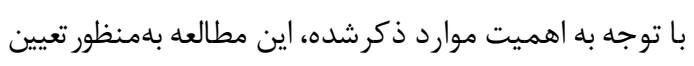

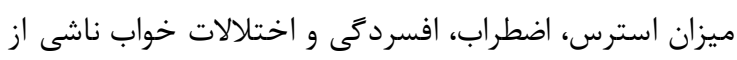

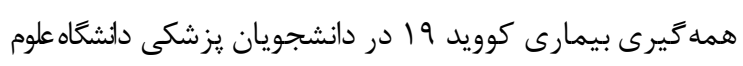

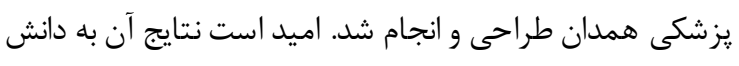

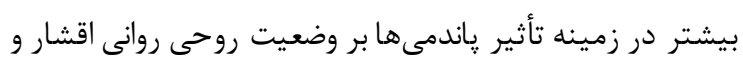

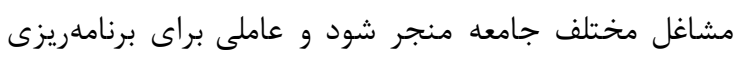

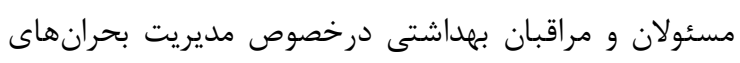
روحى ناشى از بروز اين ياندمى باشد. بهد درخد

\section{مواد و روشها}

اين مطالعه توصيفى -مقطعى پِّ از تأييد كميته اخلاق

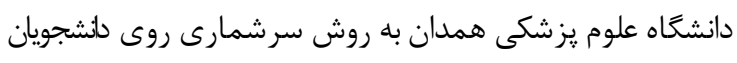

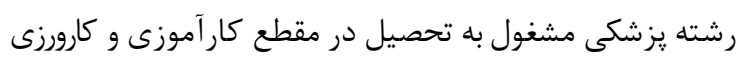

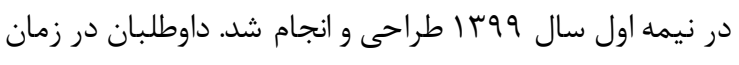

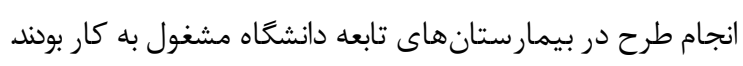

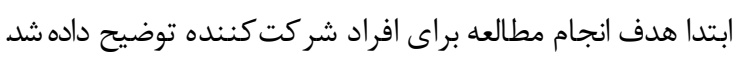

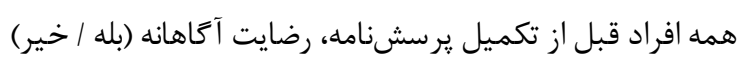

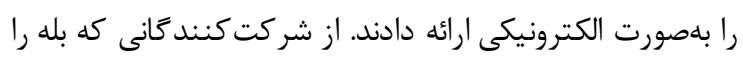


كار آموزى و بروز بى خوابى، افسردگى، اضطراب و استرس ارتباط

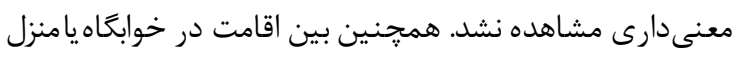

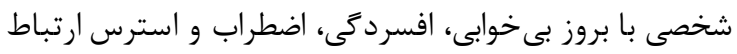

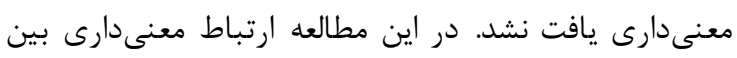

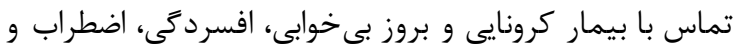

$$
\text { استرس يافت نشد (جدول س). }
$$

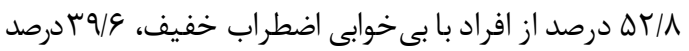

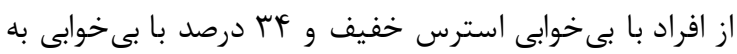

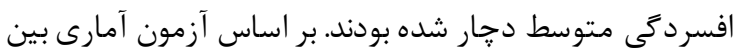

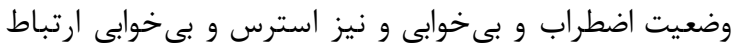

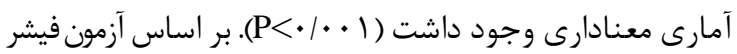

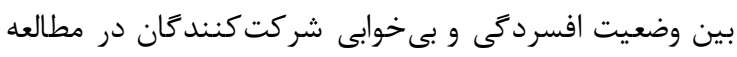

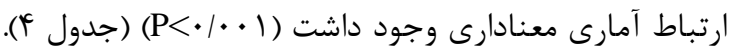

همه گيرى ويروس كرونا علاوه بر مرگتومير نسبتاً بالا و

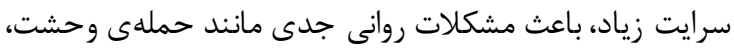

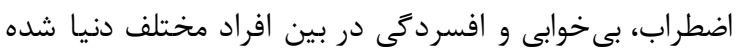
است. مطالعه حاضر تأثيرات روحى روانى همه كيرى وئى ويروس كرونا

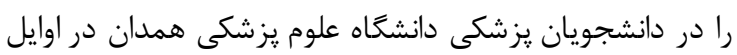

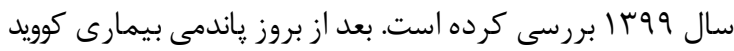

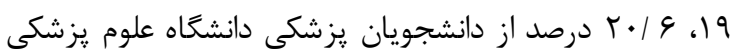

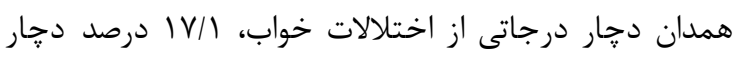

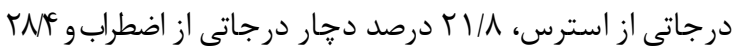

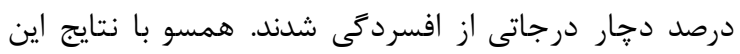

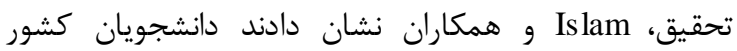

در مقياس ISI كه شامل ^ץ نمره است، شر كت كنند

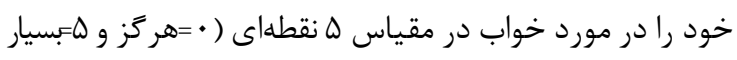

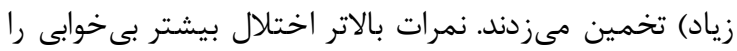

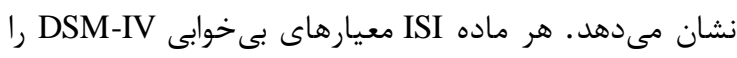
نشان مى دهد. رهنمودهاى نمرهدهى اين شاخص ازئ از اين قرهار

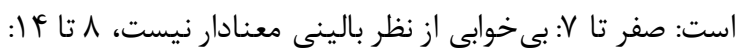

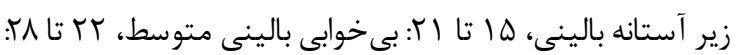
بع خوابى بالينى شديد [1 آ].

در خاتمه دادهها با استفاده از نرمافزار SPSS نسخد 19 تجزيهوتحليل شدند. براى توصيف دادههاى كمّى از ميانگين و

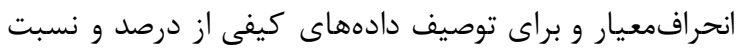

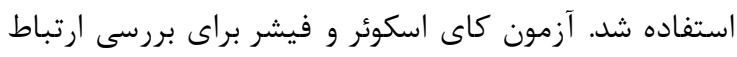

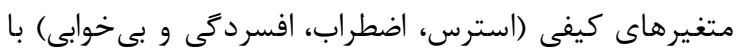

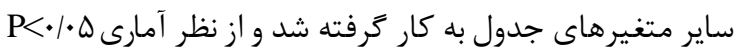

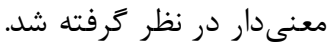

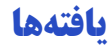

ميانگين سن شركت كنند

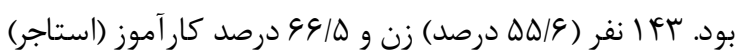
بودند. الوه درصد از شر كت كنند

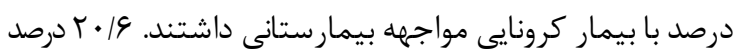

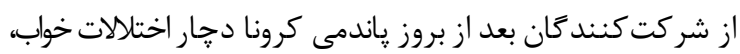

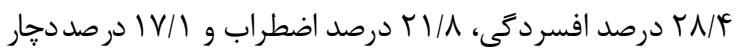

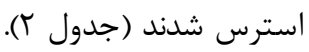

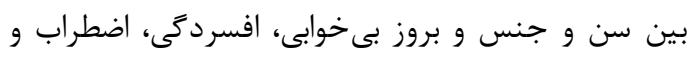

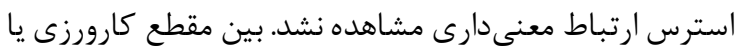

\begin{tabular}{|c|c|c|}
\hline تعداد (درصد) & (TAV= (Tداد) & 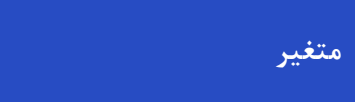 \\
\hline 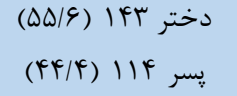 & & ? \\
\hline 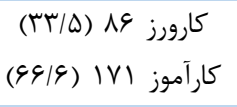 & & 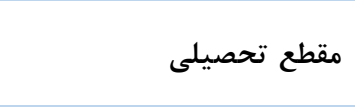 \\
\hline 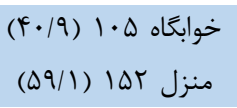 & & محل سكونت \\
\hline 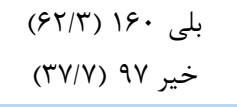 & & مواجهه با بيمار مبتلابه كرونا \\
\hline 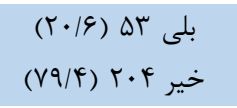 & & بى خوابى \\
\hline 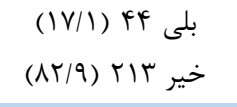 & & استرس \\
\hline 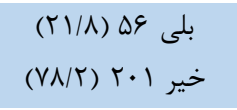 & & اضطراب \\
\hline 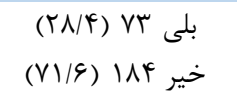 & & افسردَى \\
\hline
\end{tabular}


جدول ب: ارتباط بين مشخصات دموكرافيك و عوارض روحى روانى افراد مورد مطالعه

\begin{tabular}{|c|c|c|c|c|c|c|c|c|c|c|c|c|c|c|c|c|c|c|}
\hline \multirow{2}{*}{ ارزش P } & \multicolumn{3}{|c|}{ استر س } & \multirow{2}{*}{ ارزششP } & \multicolumn{4}{|c|}{ اضطراب } & \multirow{2}{*}{ ارزشى } & \multicolumn{4}{|c|}{ افسردىى } & \multirow{2}{*}{ ارزش P } & \multicolumn{2}{|c|}{ افسردمى } & & \multirow[b]{2}{*}{ متغير } \\
\hline & متوسط & ملايم & نرمال & & شديد & متوسط & ملايم & نرمال & & شديد & متوسط & ملايم & نرمال & & بلى & خير & & \\
\hline \multirow{3}{*}{.190} & $\begin{array}{c}r \\
(T T \pi)\end{array}$ & $\begin{array}{l}\text { If } \\
\text { (*.) }\end{array}$ & $\begin{array}{c}9 \mathrm{YV} \\
\left({ }^{*}|\Delta| \Delta\right)\end{array}$ & \multirow{3}{*}{.$\pi 4$} & $\begin{array}{c}1 \\
(1+\pi)\end{array}$ & $\begin{array}{l}\text { if } \\
(\Delta \cdot)\end{array}$ & $\hat{i}$ & $\begin{array}{c}91 \\
(f \Delta \pi)\end{array}$ & \multirow{3}{*}{$\cdot N$} & $\begin{array}{c}1 \\
(1 \cdot .)\end{array}$ & $\begin{array}{c}10 \\
\left({ }^{1} \Delta \Delta \Delta\right)\end{array}$ & $\begin{array}{c}11 \\
(\$ 9 \pi)\end{array}$ & $\begin{array}{c}\Lambda \cdot \\
(\Psi+/ \Delta)\end{array}$ & \multirow{3}{*}{$\cdot / N V$} & ${ }^{T r}$ & $\begin{array}{c}91 \\
(44), 8)\end{array}$ & . & \\
\hline & 4 & ri & 119 & & 4 & if & ir & 11. & & 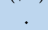 & 11 & ri & 1.4 & & $r$. & $11 \%$ & & \multirow{2}{*}{ جنس } \\
\hline & $(99 \mathrm{~N})$ & (घ.) & $(\Delta f / \Delta)$ & & $(\Lambda \Delta N)$ & $(\Delta \cdot)$ & (9/9) & $(\Delta F N)$ & & (•) & $(\Delta f / \Delta)$ & $(\Delta r / \Lambda)$ & $(\Delta \& \mid \Delta)$ & & $(\Delta 9 ; 9)$ & $\left(\Delta \Delta \wedge f^{\prime}\right)$ & j & \\
\hline \multirow{3}{*}{$\cdot M \Lambda$} & 9 & rr & Ifr & \multirow{3}{*}{$\cdot \pi$} & $r$ & 10 & if & 179 & \multirow{3}{*}{ ו } & 1 & r. & $r$ & $1 T \lambda$ & \multirow{3}{*}{$\cdot 119$} & rI & If. & استاجر & \multirow{3}{*}{ تحصيلى } \\
\hline & $(99 \mathrm{~N})$ & $(9 / / 9)$ & $(9 \mathrm{~V} / 1)$ & & (Fr/9) & $(\Delta T / 9)$ & $(99 \mathrm{~N})$ & $(99 \pi)$ & & $(1 \cdot \cdot)$ & $(9.9)$ & $(\Delta s / f)$ & $(99 / 9)$ & & $(\Delta \wedge \Delta)$ & $(\Lambda 9 / 9)$ & & \\
\hline & $\begin{array}{c}r \\
(\pi T r)\end{array}$ & $\begin{array}{c}i r \\
(\pi / / 1)\end{array}$ & $\begin{array}{l}\mathrm{V} \cdot \\
(\mathrm{Tr/q})\end{array}$ & & $\underset{(\Delta V /)}{+}$ & $\begin{array}{c}\text { ir } \\
(\& 9 / 4)\end{array}$ & 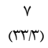 & $\begin{array}{c}9 r \\
(r \cdot(\Lambda)\end{array}$ & & $\dot{(\cdot)}$ & $\begin{array}{c}1 \pi \\
(4 a / 4)\end{array}$ & $\begin{array}{c}\text { IV } \\
(4 \pi / 9)\end{array}$ & $\begin{array}{c}\Delta \varphi \\
\left(r \cdot / f^{\prime}\right.\end{array}$ & & $\begin{array}{c}{ }^{T} \\
\left(F^{\prime} /(\Delta)\right.\end{array}$ & $\begin{array}{c}94 \\
\left(\pi / N^{4}\right)\end{array}$ & اينترن & \\
\hline \multirow{3}{*}{. NA } & 9 & $r$ & ITF & \multirow{3}{*}{ 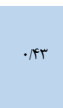 } & ${ }^{r}$ & r. & if & $11 f$ & \multirow{3}{*}{.4} & & $\pi$ & rs & 1.0 & \multirow{3}{*}{.19} & $\pi$ & 119 & منزل & \multirow{3}{*}{ سكونت } \\
\hline & $(99 \mathrm{~N})$ & (9T/9) & (QDNT) & & $(\Delta V /())$ & $(V / f)$ & $(99 \mathrm{~N})$ & $(\triangle F N)$ & & (•) & $(99 \mathrm{~N})$ & $(9+/ 1)$ & $(\Delta V / I)$ & & (9זK) & $(\Delta \Lambda N)$ & & \\
\hline & (TTK) & $(\operatorname{rV} / 1)$ & $(+) / / 1)$ & & (FT/q) & (TNG) & (זוז) & (FTr) & & $(1 \cdot)$. & (TrR) & $(\Gamma \Delta / 9)$ & $(\kappa / / 9)$ & & (TVN) & (FIN) & خوابكاه & \\
\hline \multirow{3}{*}{.19} & v & rI & ITT & \multirow{3}{*}{$\cdot / \Delta \lambda$} & 4 & IV & if & ITT & \multirow{3}{*}{$\cdot \pi$} & . & If & ro & 111 & \multirow{3}{*}{$\cdot \pi r$} & rq & $1 \pi f$ & & \multirow{3}{*}{ يموار بهه با } \\
\hline & $(\mathrm{W} / \mathrm{N})$ & (घ.) & (ST) & & $(\Lambda \Delta N)$ & $(\xi \cdot N)$ & $(99 \mathrm{~N})$ & $(g \mid \pi)$ & & (.) & $(\mathrm{TVN})$ & $(9+/)$ & $(9 \cdot \pi)$ & & (9V/9) & $(\varphi \cdot(\Lambda)$ & دارد & \\
\hline & $r^{r}$ & if & 11 & & 1 & 11 & v & VA & & 1 & 9 & if & vr & & iv & ^. & ندارد & \\
\hline \multirow{4}{*}{$\cdot \Delta r$} & $\wedge$ & $\pi r$ & $\begin{array}{l}(\pi A) \\
1 \wedge \Delta\end{array}$ & \multirow{4}{*}{$.11 \Delta$} & $\begin{array}{r}r \\
(\pi / \pi)\end{array}$ & Th & $(\pi / \pi)$ & $\begin{array}{c}\text { (TA/A) } \\
\text { IVA }\end{array}$ & & $\left(\begin{array}{l}(\cdot) \\
1\end{array}\right.$ & $\frac{T V}{T V}$ & 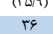 & $\begin{array}{l}(14 / 9) \\
191\end{array}$ & & (iTI) & (T/I) & زير هاT & \\
\hline & (MN9) & (91\%) & $(N Q 9)$ & & $(1 \cdot)$. & (AQT) & $\left(v / f^{\prime}\right)$ & $(M N /)$ & 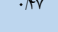 & $(1 \cdot)$. & (A)/A) & (QTא) & $(\operatorname{AV} / \Delta)$ & $\cdot(\Delta)$ & (FN9) & $(M M R)$ & سال & \\
\hline & 1 & $r$ & is & & . & $r$ & 9 & $\pi$ & & . & 9 & $r$ & $\pi$ & & $\wedge$ & if & بالاى & سن \\
\hline & $(11 / 1)$ & $(N / 9)$ & $(1 / / 1)$ & & (·) & $(1 \cdot N)$ & (TNG) & $119 / 4)$ & & (•) & $(1 M T)$ & $(\mathrm{VN})$ & $(1 Y / \Delta)$ & & $(\mid Q / /)$ & $(1 / / / 4)$ & W TH & \\
\hline
\end{tabular}

ترس از خطاهاى يزشكى در مواجها با موارد بيمارى، ترس از براز

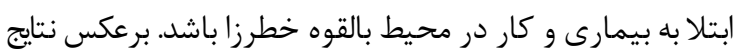

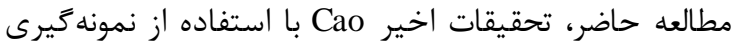

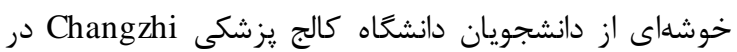

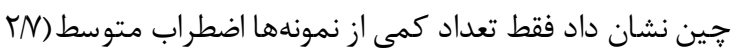

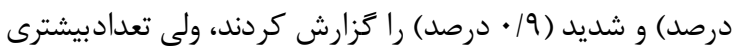

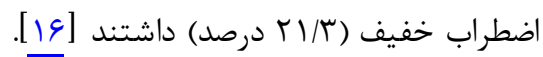

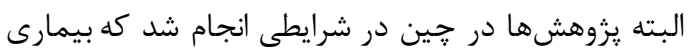
خيلى زود تحت كنترل قرار كرفت. در مطالعه انصارى و و همكاران

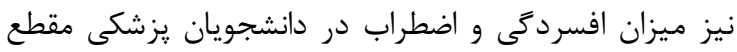

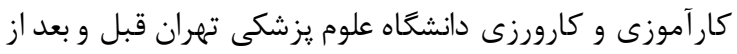

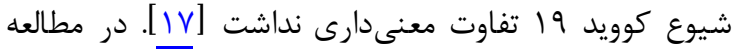

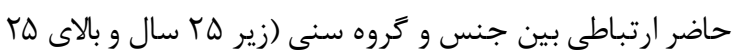

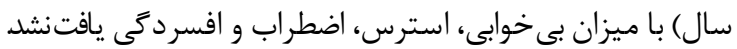

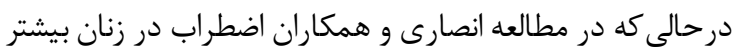

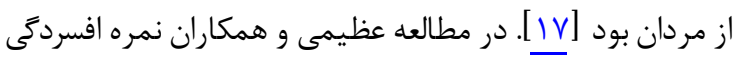

بنكلادش نيز در بحران همه كيرى كرونا با افزايش جشمخير بروز

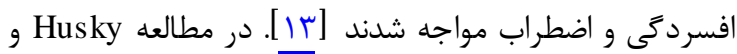

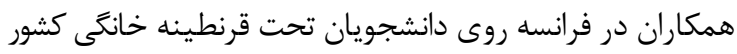

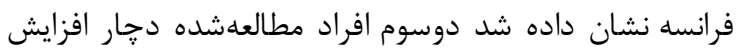

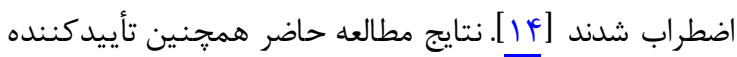

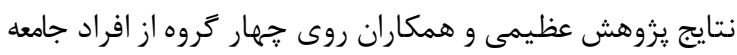

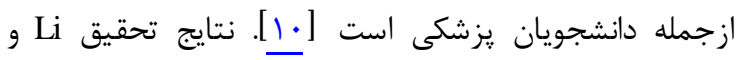

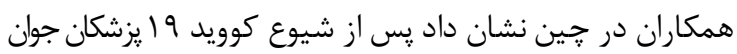

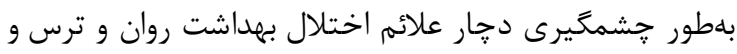

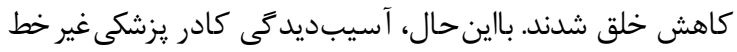

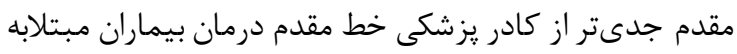
كوويد 19 بود [1] إن]

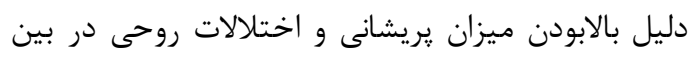

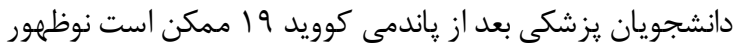

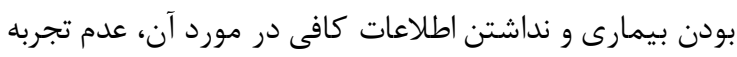

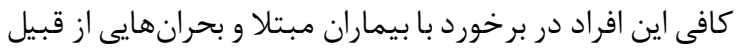

جدول fا: ارتباط بين بىخوابى باوجود و شدت اضطراب، استرس و افسردگى در افراد مطالعهشه

\begin{tabular}{|c|c|c|c|}
\hline $\mathbf{P}$ & تعداد (درصد) ندارد & تعداد (درصد) & \\
\hline$<\cdot / \cdots 1$ & $\begin{array}{c}(\Lambda F / \Lambda) \backslash V \Psi \\
(\Lambda / \Gamma) \backslash V \\
(\cdot) \cdot\end{array}$ & $\begin{array}{l}(\Delta T / \Lambda) Y \Lambda \\
(Y \cdot / \Lambda) \| \\
(I / T) V\end{array}$ & خفيف اضطراب \\
\hline$<\cdot / \cdots 1$ & $\begin{array}{c}(\Lambda F / \Lambda) \backslash V \Psi \\
(\Lambda / r) \backslash V \\
(\cdot) \cdot\end{array}$ & $\begin{array}{l}(\Delta T / \Lambda) Y \Lambda \\
(r \cdot / \Lambda) \| \\
(I / / T) Y\end{array}$ & شفيف \\
\hline$<\cdot 1 \cdot \cdot 1$ & $\begin{array}{c}(\Lambda \cdot / 9) \backslash 9 \Delta \\
(\mid \backslash / \Lambda) Y F \\
(V / F) \backslash Q \\
(\cdot) .\end{array}$ & $\begin{array}{l}(r \Delta / \Lambda) 19 \\
(r N / Y) \backslash Q \\
(Y Y) \backslash \Lambda \\
(1 / 9) \backslash\end{array}$ & شفردردى \\
\hline
\end{tabular}


Wu

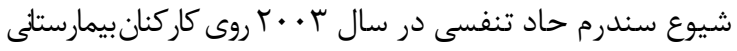

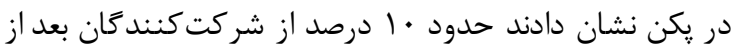
شيوع سارس سطح بالايى از علائم استرس يس إن از دان سانحه I, (PTSD: Post-Traumatic Stress Disorders) كردند و بيش از سه جهارم آنها حدود ا سال بعد هم علامتدار

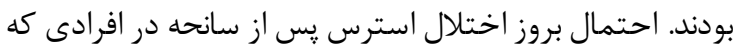

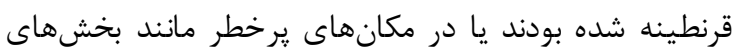

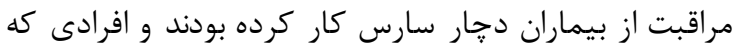

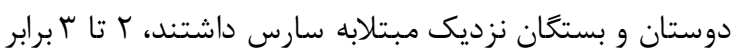

بيشتر بود [ب]. در مطالعه حاضر هY/A درصد از افراد با بى خوابى اضطراب

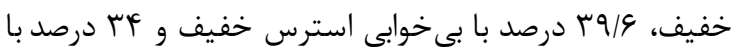
بى خوابى افسردگى متوسط داشتند و ارتباط معنى دارى بين بين

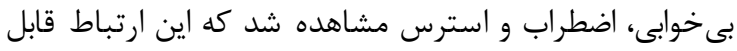

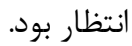

از محدوديتهاى اين مطالعه مىتوان به اين اشاره كرد كه بود

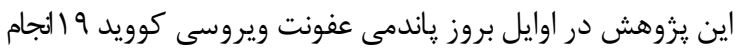

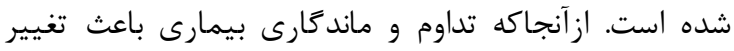

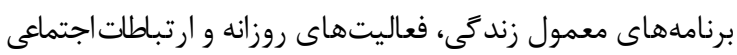

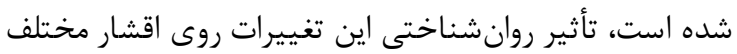

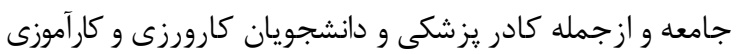

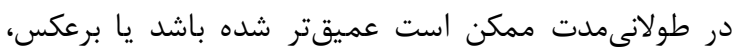

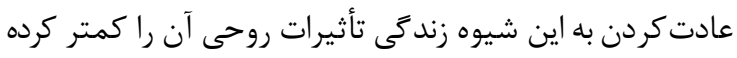

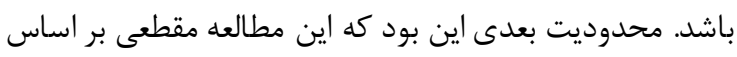

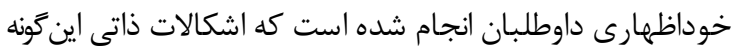

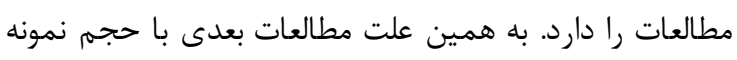

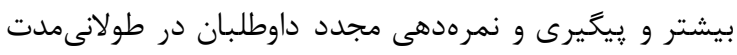
ي بيشنهاد مىشود.

\section{نتيجه كيرى}

افسردگى، اضطراب، استرس و بى خوابى در بين دانشجويان

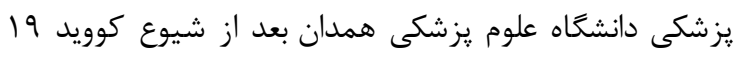

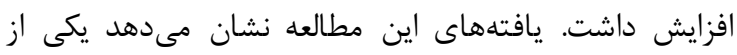

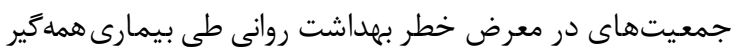

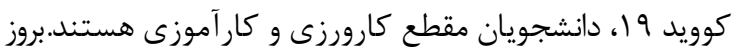
اين اختلالات ممكن است باعث كاهش توان كارى، اختلال تمركز

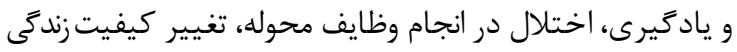

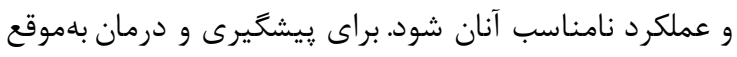

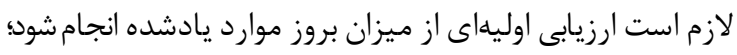

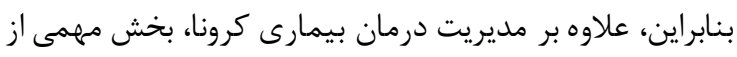

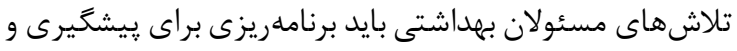

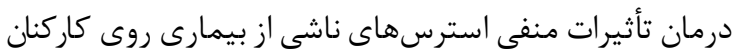

$$
\text { يزشكى در ₹ير با بيماران باشد. }
$$

در كار كنان يزشكى زن در مقايسه با مردان بيشتر ونمرهاضطراب

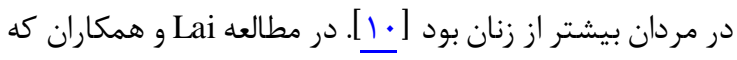

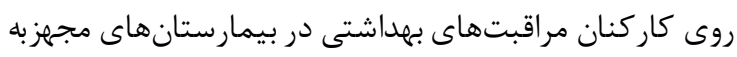
كلينيك تب و بخش هاى مخصوص بسترى بيماران مبتلابه كوويد

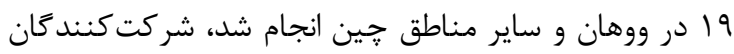

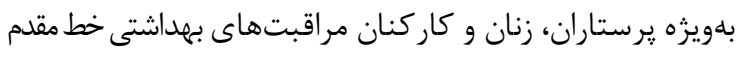
در زمينه تشخيص، درمان و مراقبت از بيماران مبتلابه كوويد 19

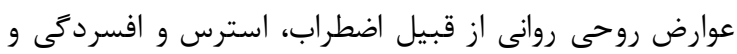
بـ خوابى را كزارش كردند [11]. در اين مطالعه بين سكونت در خوابكاه يا منزل با مشكلات

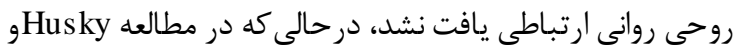

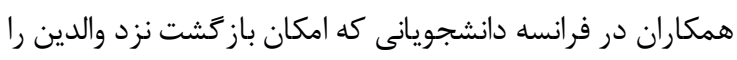

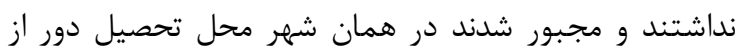

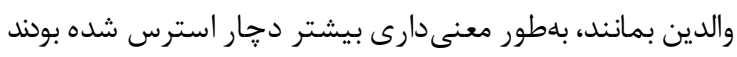

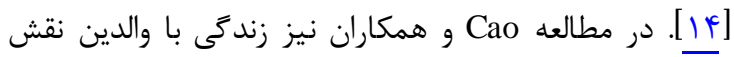

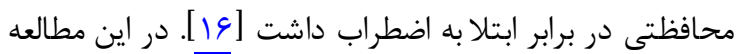

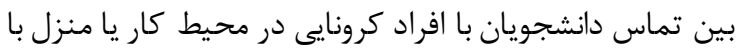
بروز مشكلات روحى و روانى ارتباطى يافت نشد. برعكس نتايج دئ داني

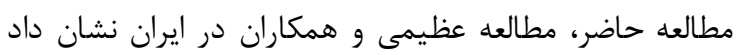
دانشجويان يزشكى كه حداقل 1 هفته تجربه كار با بيماران

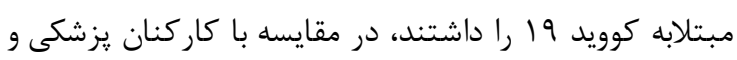

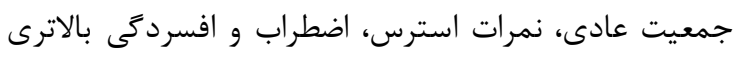

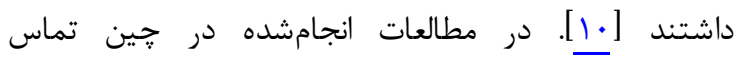
شركت كنند كان در مطالعه با افراد مبتلابه كرونا يك عامل خطر

براى افزايش بروز اضطراب بود [19 1911].

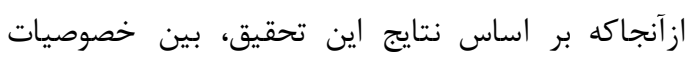

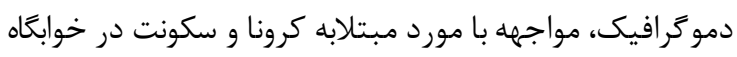

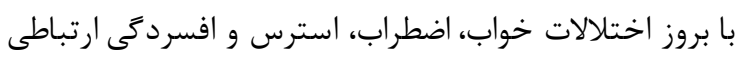

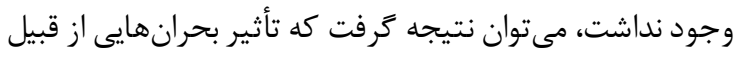

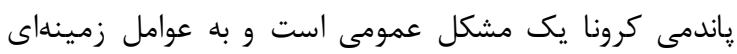

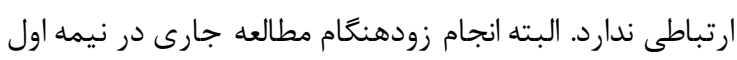

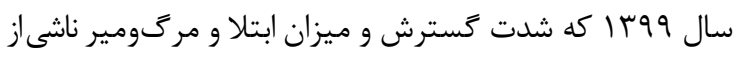

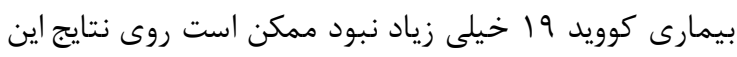

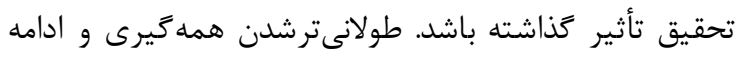

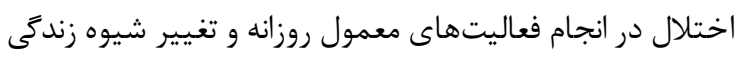

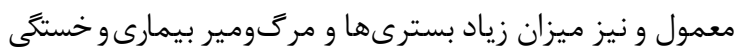

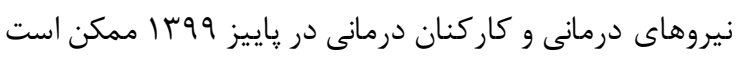

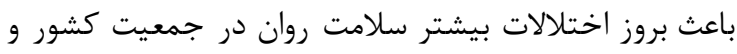

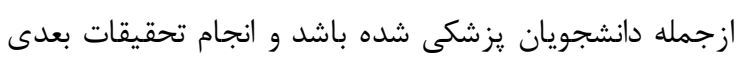

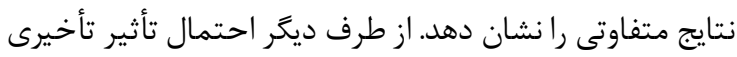

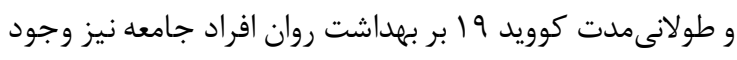

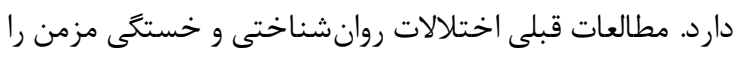
در بازماند

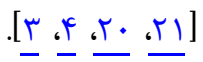


شناسه IR.UMSHA.REC.322 تأييديه دارد. همه افراد قبل

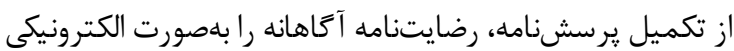

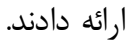

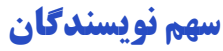

$$
\begin{aligned}
& \text { نويسنده اول (يزوهشكر اصلى): ايده انجام تحقيق، طراحى }
\end{aligned}
$$

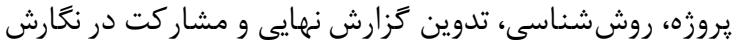

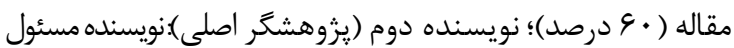

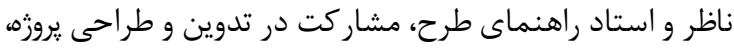

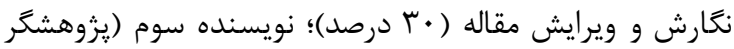

$$
\begin{aligned}
& \text { همكار): جمع آورى اطلاعات و بازنترى متون (· •ا درصد). }
\end{aligned}
$$

$$
\text { حمايت مالى }
$$

\section{REFERENCES}

1. Zhao Y, Wang P, Wu J, Wang R, Song Q. Evaluation of factors associated with anxiety and depression in chinese visiting scholars in the United States during the COVID19 pandemic assessed by online questionnaires. Med Sci Monit. 2020;26:e926602. PMID: 32966271 DOI: 10.12659/MSM.926602

2. Xiao H, Zhang Y, Kong D, Li S, Yang N. The effectsofsocial support on sleep quality of medical st aff treatingpatientswith coronavirus disease 2019 (COVID-19) in January and February 2020 in China. Med Sci Monit. 2020;26:e923549. PMID: 32132521 DOI: 10.12659/MSM.923549

3. Wu P, Fang Y, Guan Z, Fan B, Kong J, Yao Z, et al. The psychological impact of the SARS epidemic on hospital employees in China: exposure, risk perception, andaltruistic acceptance of risk. Can J Psychiatry. 2009;54(5):302-11. PMID: 19497162 DOI: $10.1177 / 070674370905400504$

4. Wu KK, Chan SK, Ma TM. Post traumatic stress, anxiety, and depression in survivors of severe acute respirat ory syndrome (SARS). J Trauma Stress. 2005;18(1):39-42. PMID: 16281194 DOI: $10.1002 /$ its. 20004

5. Hawryluck L, Gold WL, Robinson S, Pogorski S, Galea S, Styra R. SARS control and psychological effects of quarantine, Toronto, Canada. Emerg Infect Dis.2004;10(7): 1206-12.PMID: 15324539DOI: 10.3201/eid1007.030703

6. Wu KK, Chan SK, Ma TM. Post traumatic stress afterSARS Emerg Infect Dis. 2005;11(8):1297-300. PMID:16102324 DOI: 10.3201/eid1108.041083

7. Zheng W. Mental health and a novel coronavirus (2019$\mathrm{nCoV}$ ) in China. J Affect Disord. 2020;269:201-2. PMID: 32339137 DOI: $10.1016 /$ j.jad.2020.03.041

8. Wang W, Song W, Xia Z, He Y, Tang L, Hou J, et al. Sleep disturbance and psychological profiles of medical staff and non-medical staff during the early out break of COVID-19in Hubei Province, China. Front Psychiatry. 2020;11:733. PMID: 32793014 DOI: $10.3389 /$ fpsyt.2020.00733

9. Norton PJ. Depression Anxiety and Stress Scales(DASS-21): Psychometric analysis across four racial groups. Anxiety Stress Coping . 2007;20(3):253-65. PMID: 17999228DOI: 10.1080/10615800701309279

10. Vahedian-Azimi A, Moayed MS, Rahimibashar F, ShojaeiS, Ashtari S, Pourhoseingholi MA. Comparison of theseverity of psychological distress among four groups of an Iranian population regarding COVID-19 pandemic. BMCPsychiatry. 2020;20(1):402. PMID: 32770975 DOI: $10.1186 / \mathrm{s} 12888$ 020-02804-9

11. Dahm J, Wong D, Ponsford J. Validity of thedepressionanxiety stress scales in assessing depression and anxiety following traumatic brain injury. J Affect Disord. 2013;151(1): 392-6. PMID: 23830002 DOI: 10.1016/i.jad.2013.06.011

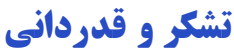

مقاله حاضر حاصل طرح تحقيقاتى مصوب كميته تحقيقات

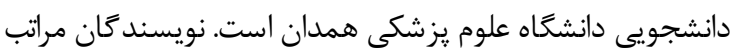

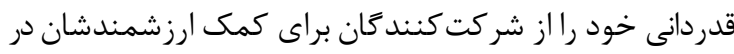
جمع آورى دادههاى مورد نياز اين مطالعه اعلام مى دارند همجهنين

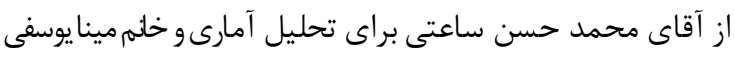

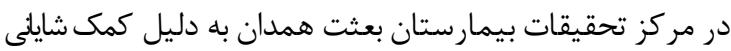

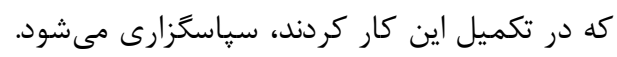

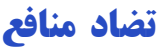

در مطالعه حاضر هيج گونه تعارض منافعى وجود نداشته

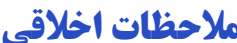 \\ اين مطالعه از كميته اخلاق دانشخاه علوم يزشكى همدان با}

12. Nojoomi M, Ghalebandi MF, Akhbari R, Gorji R. Sleep pattern and prevalence of sleep disturbances in medical students and specialist residents. Med Sci J Islam AzadUniv. 2009:19(1):55-9.

13. Islam MA, Barna SD, Raihan H, Khan MN, Hossain MT . Depression and anxiety among university studentsduringthe COVID-19 pandemic in Bangladesh: a web-based crosssectional survey. PloS One. 2020;15(8):e0238162. PMID 32845928 DOI: 10.1371 /journal.pone. 0238162

14. Husky MM, Kovess-Masfety V, Swendsen JD. Stress and anxiety among university students in France duringCovid-19 mandatory confinement. Compr Psychiatry. 2020;102: 152191. PMID: 32688023 DOI: $10.1016 /$ i.comppsych. 2020.152191

15. Li W, Frank E, Zhao Z, Chen L, Wang Z, Burmeister M, et al. Mental health of young physicians in china during the novel coronavirus disease 2019 out break. JAMA NetwOpen. 2020;3(6):e2010705. PMID: 32478846 DOI: 10.1001/ jamanet workopen.2020.10705

16. Cao W, Fang Z, Hou G, Han M, Xu X, Dong J, et al. The psychological impact of the COVID-19 epidemic oncollege students in China. Psychiatry Res. 2020;287:112934.PMID 32229390 DOI: 10.1016/j.psychres.2020.112934

17. Nakhostin-Ansari A, Sherafati A, Aghajani F, Khonji MS, Aghajani R, Shahmansouri N. Depression and anxietyamong Iranian Medical Students during COVID-19 pandemic. Iran J Psychiatry. 2020;15(3):228-35. PMID: 33193771 DOI: 10.18502/ijps.v15i3.3815

18. Lai J, Ma S, Wang Y, Cai Z, Hu J, Wei N, et al. Factors associated with mental health outcomes among health care workers exposed to coronavirus disease 2019. JAMA Netw Open. 2020;3(3):e203976. PMID: 32202646 DOI: 10.1001/jamanetworkopen.2020.3976

19. Shi L, Lu ZA, Que JY, Huang XL, Liu L, Ran MS, et al. Prevalence of and risk factors associated with ment alhealth symptoms among the general population in China during the coronavirus disease 2019 pandemic. JAMA Netw Open. 2020;3(7):e2014053. PMID: 32609353 DOI: 10.1001/jamanet workopen.2020.14053

20. Tansey CM, Louie M, Loeb M, Gold WL, Muller MP, de Jager J, et al. One-year outcomes and health care utilization in survivors of severe acute respiratory syndrome. Arch Intern Med. 2007;167(12):1312-20. PMID:17592106DOI: 10.1001/archinte.167.12.1312

21. Moldofsky H, Patcai J. Chronic widespread musculoskeletal pain, fatigue, depression and disordered sleep in chronicpostSARS syndrome; a case-controlled study. BMC Neurol. 2011;11(1):37. PMID: 21435231 DOI:10.1186/1471-2377$\underline{11-37}$ 